Imaging the Universe in Three Dimensions: Astrophysics

with Advanced Multi-Wavelength Imaging Devices.

ASP Conference Series, Vol. xxx, 2000

W. van Breugel \&5 J. Bland-Hawthorn (eds.)

\title{
SAURON: Integral-field Spectroscopy of Galaxies
}

B. W. Miller, M. Bureau, E. Verolme, P. T. de Zeeuw

Sterrewacht Leiden, Postbus 9513, 2300 RA Leiden, The Netherlands

R. Bacon, Y. Copin, E. Emsellem

Centre de Recherche Astronomique de Lyon, 9 Avenue Charles André, 69561 Saint Genis-Laval Cedex, France

R. L. Davies, R. F. Peletier, J. R. Allington-Smith

Physics Department, University of Durham, South Road, Durham, DH1 3LE, $U K$

C. M. Carollo

Dept. of Physics and Astronomy, Johns Hopkins University, $3400 \mathrm{~N}$. Charles St., Baltimore, MD 21218 USA

G. Monnet

European Southern Observatory, Karl-Schwarzschild Str. 2, D-85748

Garching, Germany

Abstract. We present the first results from a new and unique integralfield spectrograph, SAURON. Based upon the TIGER concept, SAURON uses a lens array to obtain two-dimensional spectroscopy with complete spatial coverage over a field of $33^{\prime \prime} \times 41^{\prime \prime}$ in low-resolution mode $(0.94$ lenslets) and of $9^{\prime \prime} \times 11^{\prime \prime}$ in high-resolution mode ( 0 '.26 lenslets). The spectra cover the wavelengths from $4800 \AA$ to $5400 \AA$ with a spectral resolution of $\sim 3 \AA\left(\sigma \approx 75 \mathrm{~km} \mathrm{~s}^{-1}\right)$. SAURON achieved first light during commissioning on the William Herschel Telescope on 1 February 1999. We are now commencing a systematic survey of the velocity dispersions, velocity fields, and line-strength distributions of nearby ellipticals and spiral bulges. The wide field of SAURON will be crucial for unraveling complicated velocity structures. In combination with available long-slit spectroscopy of the outer regions of the galaxies, HST spectra of the nuclei, HST imaging, and dynamical modeling, we will constrain the intrinsic shapes, mass-to-light ratios, and stellar populations in spheroids.

\section{Introduction}

Understanding the formation and evolution of elliptical galaxies and spiral bulges is complicated by the fact that many, if not all, of these systems are triaxial or have multiple kinematic components. Both lead to rich velocity structures 
that are difficult to map using long-slit spectroscopy. In addition, the central few arcseconds of spheroids are often "kinematically decoupled": the inner and outer regions appear to rotate around different axes (e.g., Illingworth \& Franx 1989). This makes two-dimensional (integral-field) spectroscopy of stars and gas essential for determining the dynamical structure of these systems. Therefore, the galactic dynamics groups in Lyon, Leiden, and Durham decided to build SAURON, a unique integral-field unit (IFU) with a large field of view and high throughput that is optimized for studies of the gaseous and stellar kinematics in galaxies.

\section{The SAURON Instrument}

SAURON (Spectroscopic Areal Unit for Research on Optical Nebulae) is a TIGERlike integral field spectrograph (Bacon et al. 1995) currently being used at the 4.2-m William Herschel Telescope of the Isaac Newton Group on La Palma. The focal plane is sampled by an array of 1520 square lenses whose projected size on the sky can be set to $0^{\prime \prime} \cdot 94$ or $0^{\prime \prime} \cdot 26$, yielding fields of view of $33^{\prime \prime} \times 41^{\prime \prime}$ or $9^{\prime \prime} \times 11^{\prime \prime}$, respectively. About 70 dedicated lenses sample an area offset 1 .'7 from the main field, to allow sky subtraction. The spatial coverage is $100 \%$. SAURON complements OASIS on the CFHT, which allows high spatial resolution sampling of the nuclear regions (e.g., one can obtain a 4 ". $1 \times 3$ "'.3 field with 0 "' 11 sampling for use with the Adaptive Optics Bonnette).

The grism is optimized for the spectral domain $4800-5400 \AA$, which includes important absorption/emission lines such as the $\mathrm{Mg}$ triplet, Fe lines, $\mathrm{H} \beta$, [OIII $] \lambda 4959,5007$, and [NI $] \lambda 5199$. The instrumental velocity dispersions are $82 \mathrm{~km} \mathrm{~s}^{-1}$ (0'.94 sampling) or $\sim 64 \mathrm{~km} \mathrm{~s}^{-1}$ (0'.26 sampling).

SAURON was commissioned on the WHT 1-5 February 1999. The instrument performed admirably. Most of the effort went to focusing the optics, aligning the CCD, grism, and lenslet array, and adjusting the baffling to remove stray light and reflections. To prevent the spectra from overlapping, the angle between the grism and the lenslet array was set to 5.19. Flexure was found to be less than 1 pixel in both the spatial and spectral directions in $\sim 1800$ seconds.

\section{The SAURON Project}

During the project we hope to observe about 80 bright galaxies covering all Hubble types. Initially, we are concentrating on E, S0, and Sa types. We are restricting the sample to bright $\left(M_{B}<-18\right)$ northern galaxies with $v_{\odot}<3000 \mathrm{~km} \mathrm{~s}^{-1}$ and available HST imaging and/or spectroscopy. This will be a representative sample covering the full parameter space of absolute magnitude, effective radius $\left(R_{\mathrm{e}}\right)$, flattening, rotational support, and $\mathrm{Mg}_{b}$ index.

We require $S / N>25$ per resolution element to map the velocity fields and $S / N>40$ to measure accurate velocity profiles. Exposure times of $\sim 2$ hours per position are needed to reach these levels at $\mu_{B} \approx 20$ mag $\operatorname{arcsec}^{-2}$. We also aim to map as much of the inner $1 R_{\mathrm{e}}$ as possible, using multiple pointings if necessary. 


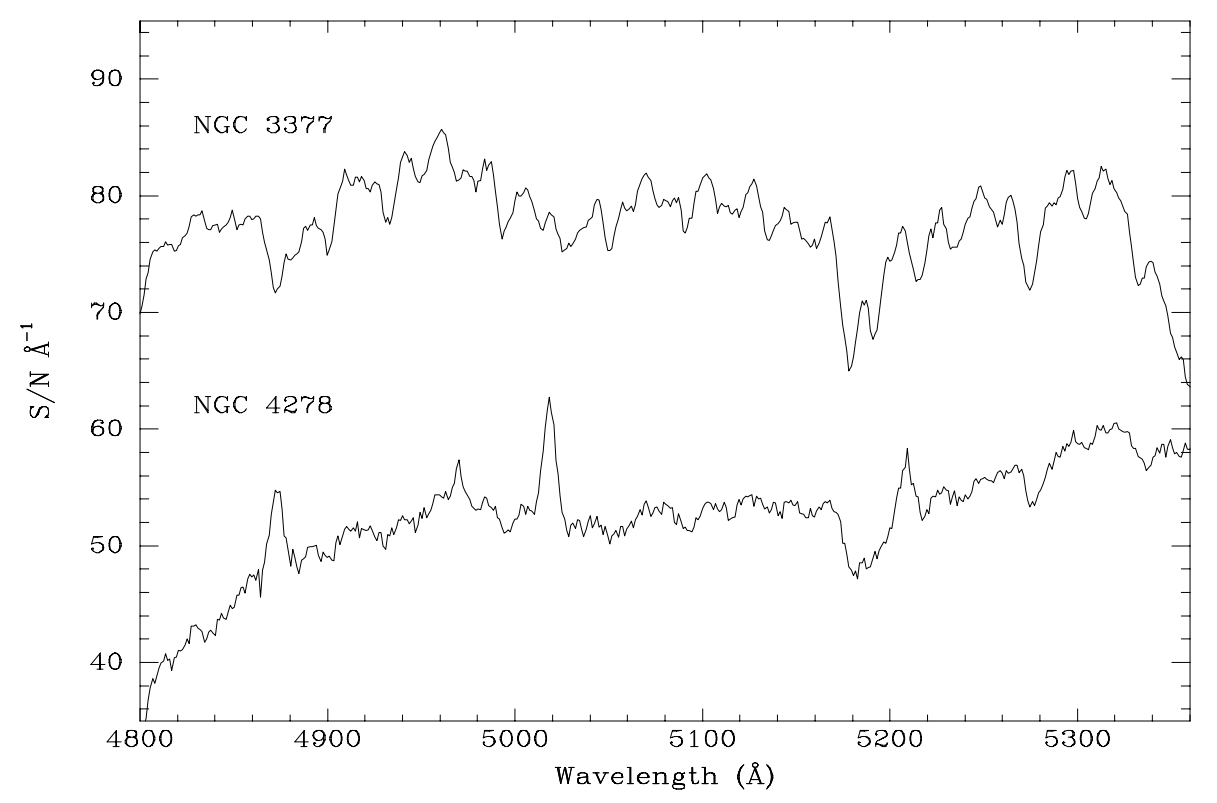

Figure 1. Example SAURON spectra for NGC 3377 and NGC 4278. The NGC 3377 spectrum shows absorption lines from $\mathrm{H} \beta, \mathrm{Mg}$, and Fe. NGC 4278 has lines of $\mathrm{H} \beta$, [OIII], and [NI] in emission as well as $\mathrm{Mg}$ and $\mathrm{Fe}$ in absorption.

The first science run was 14-21 February, 1999. Figure 1 shows preliminary SAURON spectra from the centers of NGC 3377 and NGC 4278. These spectra were made by summing a strip 3 pixels wide perpendicular to the dispersion direction. The sky has been subtracted but no flat-fielding has been attempted. SAURON can reach $S / N \approx 65 \AA^{-1}$ in 1800 seconds in high surface brightness regions. Emission and absorption lines from $\mathrm{H} \beta$, [OIII], $\mathrm{Mg}_{b}$, [NI], and FeI are visible.

The real advantage of SAURON is the ability to produce maps of velocities, velocity dispersions, and line strengths. Figure 2 a shows a preliminary velocity field for NGC 3377. It reveals a rotating disk with a maximum velocity of $\sim 80 \mathrm{~km} \mathrm{~s}^{-1}$. The velocity dispersion (Figure $2 \mathrm{~b}$ ) is lower in the outer parts of the disk and then increases towards the nucleus. Line-strength maps indicate increasing metallicity towards the center (Davies 1999)

We are currently in the process of refining the data reduction procedure. The method will be similar to that used to reduce and analyze OASIS data from the CFHT. Tight packing of the SAURON spectra means that the extraction process must be precise. To account for contamination from neighboring spectra, we employ an optimized method that models the distortion of the optics and fits a PSF at each wavelength of each spectrum. We are also developing a data reduction pipeline and a data archive.

The data will be compared with fully general numerical galaxy models built with Schwarzschild's orbit superposition method. All appropriate imaging and spectral data will be used as input for the models: high resolution HST spectra 


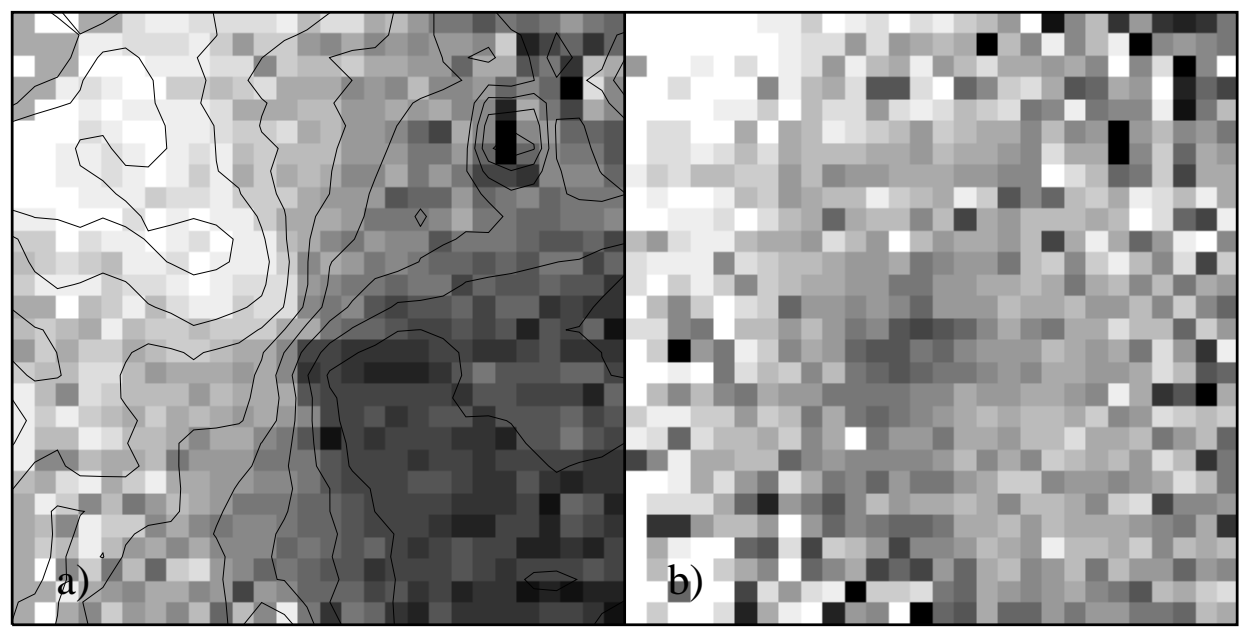

Figure 2. Preliminary SAURON maps of NGC 3377. Each panel shows a field $26^{\prime \prime}$ on a side. a) Stellar velocity field, showing evidence for rotation. The contour interval is $20 \mathrm{~km} \mathrm{~s}^{-1}$. b) Stellar velocity dispersion map, indicating an increase in $\sigma$ towards the nucleus.

for constraining the mass of the central black hole; SAURON data cubes for unraveling complicated velocity structures of the stars and gas within $1 R_{\mathrm{e}}$; longslit spectra for detecting the presence of dark matter halos at $(1-2) R_{\mathrm{e}}$; and HST images and SAURON line-strength maps for estimating the structure and $M / L$ of the starlight. The galaxy models make no assumptions about velocity anisotropy and account for PSF convolution and the finite size of the spatial element (i.e., microlens). It has been applied successfully to determine black hole masses in the E3 galaxy M32 (van der Marel et al. 1998) and in NGC 4342, an E7/S0 in Virgo (Cretton \& van den Bosch 1999).

The output of the SAURON project will be a large, uniformly processed and analyzed sample. Such a dataset is essential for understanding the formation and evolution of elliptical galaxies, spiral bulges, and bars.

We would like to thank the Dutch and UK PATTs for allocation of time on the WHT and the ING staff for their support during the SAURON commissioning and science runs. B.W.M. also thanks the Leids Kerkhoven Bosscha Fonds for financial support to attend this conference.

\section{References}

Bacon, R., Adam, G., Baranne, A., Courtès, G., Dubet, D., Dubois, J. P., Emsellem, E., Ferruit, P., Georgelin, Y., Monnet, G., Pécontal, E., Rousset, A., \& Sayède, F. 1995, A\&AS 113,347

Cretton, N., \& van den Bosch, F.C., 1999, ApJ, 514, 704

Davies, R. 1999, this volume

Illingworth, G. D., \& Franx, M. 1989, in Dynamics of Dense Stellar Systems (Cambridge), 13 van der Marel R.P., Cretton, N., de Zeeuw, T., \& Rix, H.W. 1998, ApJ, 493, 613 